IF-TU-22

\title{
Traveling-Wave Tube Amplifier Performance Evaluation and Design Optimization for Applications in Multi-level Digital Communications
}

\author{
J. Qiu ${ }^{(a)}$, D. Abe ${ }^{(a)}$, T.M. Antonsen, Jr ${ }^{(b)}$, B.G. Danly ${ }^{(a)}$ and B. Levush ${ }^{(a)}$ \\ (a) Naval Research Laboratory, Washington, DC, 20375, USA \\ (b) University of Maryland, College Park, MD, 20742, USA
}

\begin{abstract}
In this paper we use power margin as a figure of merit for evaluating the performance of TWT amplifiers used in multi-level digital communications applications. Power margin is better at predicting the system level performance of TWT amplifiers than device-level measures like EVM. We compare the calculated power margin performances for helix TWT circuits optimized with different optimization goal functions in CHRISTINE. A digital goal function that provides enhanced power margin is constructed to demonstrate the approach of TWT circuit design optimization from a system perspective.
\end{abstract}

\section{INTRODUCTION}

The increasing need for high data rate (HDR) links in military and commercial communication systems, coupled with the limitations on available spectrum, is resulting in renewed interest in high spectral efficiency modulations. For the designer of power amplifiers to be utilized in these new systems, the use of multi-level modulations places a stringent requirement on power amplifier linearity. Whether one is concerned with the battery life of a cell phone with a solid state MMIC amplifier or the power budget in a satellite communication system employing a traveling-wave tube amplifier (TWTA), the system objective is the same, namely, maximizing link margin while minimizing prime power requirements. For the device perspective, this translates to an objective of minimizing distortion while maximizing device efficiency. The design of power amplifier to optimally achieve this objective is the subject of much work. This paper demonstrates a procedure for the optimal design of a TWTA using the power margin on a communications link as the figure of merit. It is shown that the optimization procedure can be used not only for setting the operating point of the TWTA but also for optimization of the physical design of the TWT itself. The use of TWTAs designed by such a procedure can in principle yield $\mathrm{a}^{\text {! }}$ substantial improvement in the system link margin over systems employing TWTAs designed according to more traditional analog distortion specifications. In this paper, we will concentrate on applications where the bandwidth of the digital modulated signal being amplified by the
TWTA is relatively narrow so that the gain is independent of frequency and the memoryless nonlinear model [1][2] for the TWT can be applied.

In these types of applications, two issues are of great importance. The first is, for an existing TWTA, the determination of optimal drive level. The conflicting requirements of efficiency and linearity prevent the TWTA from operating near saturation and operating the TWTA with low efficiency would be economically undesirable. From the system perspective, optimal operation of a TWTA requires the tradeoff between power and distortion. The second issue is the optimization of the TWT design to achieve the highest possible performance. Dynamic velocity tapering (DVT) is a well-known technique to improve the efficiency and linearity of TWTs by properly varying circuit physical parameters, such as helix pitch profiles [3]. With the recent advances in high fidelity computer modeling of TWTs and the rapid increase in computing speed, it is possible to quickly evaluate TWT circuits and perform iterative optimizations by changing circuit parameters. The physics-based helix TWT design code, CHRISTINE, developed by NRL and SAIC is one example of a modern high fidelity TWT design code [4]. Traditional performance specification of TWT like $\mathrm{AM} / \mathrm{AM}$ and $\mathrm{AM} / \mathrm{PM}$ distortion provides only limited insight in addressing the two issues. System level figures of merit are therefore needed for such investigation.

In section 2, a memoryless nonlinear model of the TWT is described. In section 3, we calculate the power margin performance of a TWTA using 16-QAM modulation. This modulation has been chosen as representative of a multilevel modulation which would require significantly better linearity in the power amplifier (PA) than more traditional near constant-envelope modulations (MSK, BPSK, QPSK). In section 4, we make use of CHRISTINE's optimization capabilities to design optimized helix TWT circuits. The power margin for the optimized TWT circuits are compared. The goal functions used include AM/PM minimization, complex gain linearization, maximum efficiency maximization and a new digital goal function that provides optimized power margin. 


\section{MEMORYLESS NONLINEAR MODEL FOR TWTA}

If the bandwidth used in a modulated carrier communication system is small enough such that the amplifier transfer characteristic is essentially frequency independent over the bandwidth of the signal, the amplifier is traditionally modeled by the instantaneous nonlinear. amplitude and phase conversion functions.

Assume the input signal is given by,

$$
x(t)=A(t) \cos \left[2 \pi f_{0} t+\phi(t)\right]
$$

where $f_{0}$ is carrier frequency. The digital information is carried by $A(t)$ and $\phi(t)$ whose exact forms depend on the modulation formats. The output through a memoryless nonlinearity can be written as,

$$
y(t)=f[A(t)] \cos \left\{2 \pi f_{0} t+\phi(t)+g[A(t)]\right\}
$$

where $f(A)$ is the nonlinear gain conversion and $g(A)$ is the nonlinear phase conversion. The experimental nonlinear conversion curves for a Hughes $8573 \mathrm{H}$ helix TWTA at $1.65 \mathrm{GHz}$ are shown in Fig. 1.

\section{PERFORMANCE EVALUATION OF TWTA}

For low order modulations like BPSK and QPSK, spectral regrowth and cross channel interference are the dominant factors in determining the operating point of a TWTA. However, for higher order modulations like $\mathrm{m}$ QAM modulation, other factors, such as power margin discussed below, become more sensitive to nonlinear distortion and therefore dictate the amplifier operating point.

The probability of demodulation error at the receiver is typically the prime performance measure in digital communication systems. A demodulation error occurs when the demodulated location of a signal falls outside of the decision boundary of its associated reference constellation. When the received signal is corrupted by additive white Gaussian noise, the symbol-error-rate (SER) can be estimated by integrating the portion of a 2-D Gaussian distribution outside the decision boundary of a particular reference. The origin of the Gaussian distribution is the location of the demodulated signal without the noise. The locations of the demodulated signals will not coincide with the rectangular reference except in the linear case. In order to achieve the same SER, higher carrier-to-noise ratio (CNR) is needed with increasing input drive. This is because the nonlinear distortion causes the demodulated signals to move away from the references and closer to the decision boundaries. This increases the probability for errors to occur. In order to compensate for this, higher output power is needed to push the references away from each other to achieve the
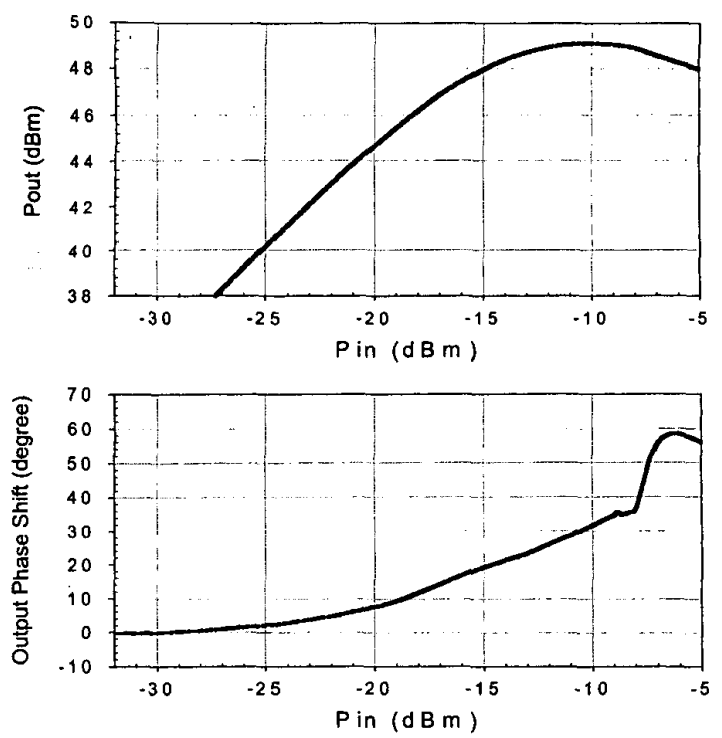

Fig. 1. Nonlinear Gain Conversion and Nonlinear Phase Conversion Functions for the Hughes $8573 \mathrm{H}$ Helix TWTA at $1.65 \mathrm{GHz}$.

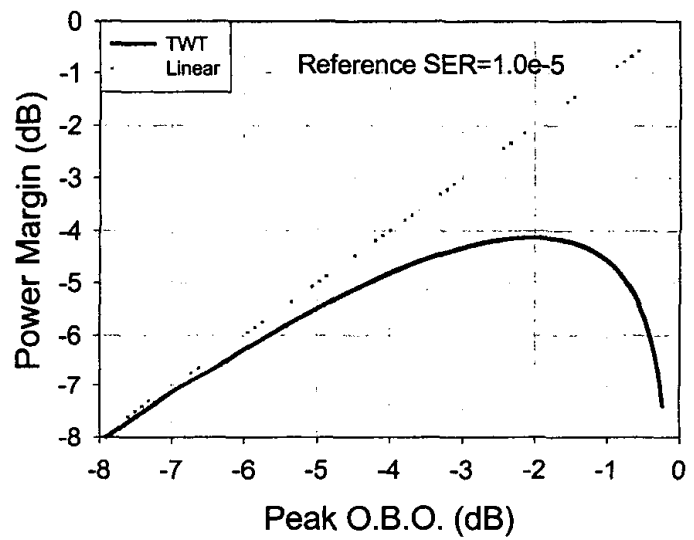

Fig. 2. Power Margin for 16-QAM Plotted against Peak Output Back-off (O.B.O.) from Saturation.

same SER level. Such an increase in required power and therefore increase in CNR represents a power penalty for operating an amplifier in its nonlinear regime. The optimized operation of TWTA represents a balance between the available output power and the power penalty due to nonlinear distortion.

Power margin is defined as the ratio between the available power and the power needed to sustain a particular SER level with a known noise level. The inverse of power margin is also referred to as the total degradation 

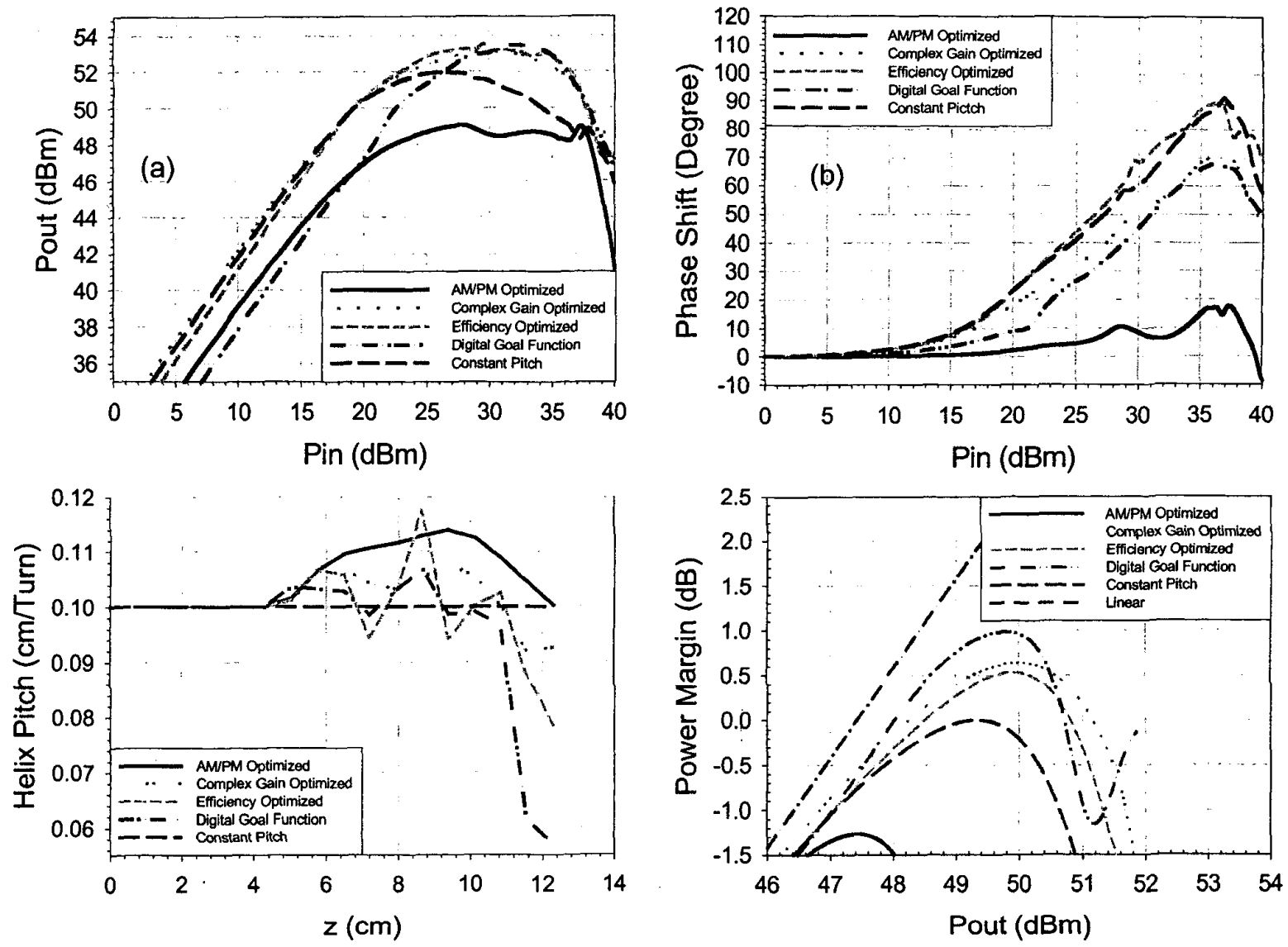

Fig. 3. (a) and (b) Drive Curves for the Optimized Circuits.

in the literature. Power Margin analysis is often used to optimize power amplifier operating point [5].

The absolute value of the power margin depends on a number of factors: antennas, path losses and noise levels, etc, which are not the concern of this paper. The important features of the power margin curve remain unchanged as long as the nonlinearity is fixed. Therefore the power margin curve can be very useful for setting the optimal operating point for an existing TWTA as well as comparing the relative performance of different TWT designs.

In Fig 2, we plot the power margin for the Hughes $8573 \mathrm{H}$ TWTA at $1.65 \mathrm{GHz}$ for $16-\mathrm{QAM}$ signals. The power margin is normalized to the achievable power margin for a linear amplifier with peak output power equal to saturation power of the TWTA. The curve indicates that the TWTA's optimal operation requires a $2 \mathrm{~dB}$ output back-off with a maximum achievable power margin about $4 \mathrm{~dB}$ lower than an ideal linear amplifier with the same maximum available power. The reference constellations used in the calculation are generated by requiring that,

$$
\frac{1}{M} \sqrt{\sum_{i=1}^{M}\left|s_{i}-\lambda z_{i}\right|^{2}}
$$

is minimized, where $M$ is the modulation order, $s_{i}$ is the recovered symbols, $z_{i}$ is the ideal constellation reference and $\lambda$ is a complex scaling factor.

\section{TWT CIRCUIT DESIGN OPTIMIZATION}

Power margin also provides a mean for comparing the performance of TWT circuit designs.

The TWT design code, CHRISTINE, has the capability of optimizing circuit parameters to achieve desired performance characteristics depending on the specified goal functions. The circuit parameters that can be varied are those describing the circuit (pitch profile, helix radius, vane radius, etc.). In this section, we compare the power margin performance for 4 circuit designs for a C-band 
helix TWT at $5 \mathrm{GHz}$ optimized with 4 different goal functions. The Goal functions are:

1. AM/PM Optimization:

$$
\text { Error }_{\text {phase }}=\int_{0}^{P_{\max }} \frac{d P_{\text {out }}}{P_{\max }}\left|\phi\left(P_{\text {in }}\right)-\phi(0)\right|
$$

is minimized, where $\phi\left(P_{i n}\right)$ is the output phase shift and $P_{\max }$ is a specified maximum output power.

2. Complex gain optimization:

$$
\text { Error }_{\text {gain }}=\int_{0}^{P_{\max }} \frac{d P_{\text {out }}}{P_{\max }}\left|\frac{g\left(P_{\text {in }}\right)-g(0)}{g(0)}\right|
$$

is minimized, where $g\left(P_{i n}\right)$ is the complex gain.

3. Efficiency optimization:

$$
\eta=\frac{P_{\text {out }}-P_{\text {in }}}{I V}
$$

is maximized, where $I$ and $V$ are the beam current and voltage, respectively.

4. Digital optimization:

$$
\begin{aligned}
& \text { Error }_{\text {digital }}=\bar{P}_{\text {out }}(d B) \\
& -\log _{10}\left[\left(\frac{D}{d_{1}}\right)^{20}+\left(\frac{D}{d_{2}}\right)^{20}+\left(\frac{D}{d_{3}}\right)^{20}+\left(\frac{D}{d_{4}}\right)^{20}\right]
\end{aligned}
$$

is maximized, where $\bar{P}_{\text {out }}$ is the average power of the recovered symbols at the moment of symbol decision, $D$ is the distance from one of the reference constellation to its decision boundary, $d_{1}$ is the shortest distance for all the recovered symbols to their decision boundaries in the first quadrant of the $\mathrm{I} / \mathrm{Q}$ plane, $d_{2}$ is the second shortest distance, and so on.

Goal functions 1, 2 and 3 have traditionally been used in optimizing TWT designs for analog applications. Their definitions do not contain digital modulation information, so it is unlikely for them to result in designs that produce an optimal power margin. The definition of power margin requires the simultaneous optimization of efficiency and linearity. Goal function 4 is a loose approximation of power margin and should produce that most power margin. It is motivated by the principle of union bond [6][7].

In each of the optimized designs, helix profiles are varied while all other circuit parameters are held fixed to achieve the desired optimization. The electron beam current and voltage therefore beam power are also fixed. The characteristics for each of the circuits are shown in Fig 3. The drive curves are shown in Fig. 3(a) and (b), the helix pitch profile in (c) and the power margin in (d). The power margin curves are calculated by estimating the SER using the drive curves in (a) and (b). The fact that circuit optimized with the digital goal function has the highest power margin and achieves $1 \mathrm{~dB}$ improvement in power margin compared to the unoptimized, constant pitch case demonstrates that it is possible to optimize a TWT circuit according to system level information like modulation format, communication standards and power margin, etc.

\section{CONCLUSION}

In this paper a procedure for optimization of TWT physical design based on power margin has been examined. Although the established traditional figures of merit such as $\mathrm{AM} / \mathrm{PM}$ conversion and complex gain can lead to TWTA designs performing better than untapered helix designs as expected, the use of digital-modulationspecific power margin as a figure of merit has been shown to lead to further improvement in TWTA designs. This example suggests that improved TWTA design for new spectrally efficient modulations can yield significant improvements in link margins for new communication systems

\section{ACKNOWLEDGEMENT}

The authors would like to thank Drs. J.P. Calame and P. Safier for useful discussions. The loan of the $8573 \mathrm{H}$ TWTA by Boeing Electron Dynamic Devices for experimental tests is greatly appreciated. This work is supported by the Office of Naval Research.

\section{REFERENCES}

[1] M.C. Jeruchim, P. Balaban and K.S. Shanmugan, Simulation of Communication Systems, New York: Plenum Press, 1992.

[2] A.A.M Saleh, "Frequency-Independent and FrequencyDependent Nonlinear Models of TWT Amplifier," IEEE Transactions on Communications, vol. COM-29, no. 11, pp. 1715-1720, November, 1981.

[3] A.S. Gilmour, Jr., Principles of Traveling Wave Tubes, Boston: Artech House, 1994.

[4] T.M. Antonsen, Jr. and B. Levush, "CHRISTINE: $A$ Multifrequency Parametric Simulation Code for Travelingwave Tube Amplifiers," NRL Rep. 97-9845, 1997.

[5] S. Pupolin and L.J. Greenstein, "Performance Analysis of Digital Radio Links with Nonlinear Transmit Amplifiers," IEEE Journal on Selected Areas in Communications, vol. SAC-5, no. 3, pp. 534-546, April 1987.

[6] J.G Proakis, Digital Communications, New York: McGraw Hill, 2000.

[7] J.W. Craig, "A New, Simple and Exact Result for Calculating the Probability of Error for Two-dimensional Signal Constellations," IEEE Military Communications Conference, pp. 571-575, 1991 